\title{
REVIEW
}

\section{Management of ejaculatory disorders in infertile men}

\author{
Yagil Barazani ${ }^{1}$, Peter J Stahl ${ }^{2}$, Harris M Nagler ${ }^{1}$ and Doron S Stember ${ }^{1}$ \\ Ejaculatory dysfunction is a highly prevalent clinical condition that may be classified along a continuum that ranges from premature \\ ejaculation (PE), through retarded or delayed ejaculation (DE), to complete anejaculation (AE). Retrograde ejaculation (RE) represents \\ a distinct entity in which ejaculate is expelled either partially or completely into the bladder. While DE and PE are significant sources of \\ sexual dissatisfaction among men and their partners, patients with these disorders retain normal fertility in most cases. Conversely, \\ men with $A E$ and RE are unable to deliver sperm into the female genital tract and are therefore rendered subfertile. Therefore, in \\ reviewing ejaculatory disorders as they relate to fertility, this paper will primarily focus on the diagnosis and management of $A E$ and $R E$. \\ Physiology, diagnostic strategies, pharmacological treatments, and procedural interventions relevant to AE and RE are discussed. \\ Asian Journal of Andrology (2012) 14, 525-529; doi:10.1038/aja.2012.29; published online 14 May 2012
}

Keywords: anejaculation; delayed ejaculation; ejaculatory dysfunction; electroejaculation; penile vibratory stimulation; premature ejaculation; retrograde ejaculation

\section{INTRODUCTION}

Disorders of ejaculation can be classified along a spectrum ranging from premature ejaculation (PE), through retarded or delayed ejaculation (DE), to complete anejaculation (AE) along with retrograde ejaculation (RE). Ejaculatory dysfunction (excluding PE) interferes with delivery of sperm to the female genital tract and is an important and, often under-appreciated, etiological factor in male subfertility. In a multinational survey of more than 11000 men capable of achieving erections, ejaculatory problems (defined as a reduced amount of ejaculate or a complete absence of ejaculation) were reported by $30.1 \%, 54.9 \%$ and $74.3 \%$ of men aged $50-59,60-69$ and $70-80$ years, respectively. Reduced amount of ejaculation and complete absence of ejaculate within the previous month were reported by $46 \%$ and $5 \%$ of respondents, respectively. This ejaculatory dysfunction was associated with bother independent of age, ranging from $50 \%$ to $59 \%$ across all age groups. ${ }^{1}$

DE is a common cause of sexual frustration. Men with DE have difficulty in achieving orgasm and, in some cases, give up before reaching it. PE represents another common cause of dissatisfaction in which ejaculation occurs distressingly soon after, or even prior to, penetration or stimulation., ${ }^{2,3}$ While DE and PE are significant sources of sexual dissatisfaction among men and their partners, ${ }^{4}$ these disorders are not commonly associated with infertility. Men with AE and RE, on the other hand, are by definition unable to deliver sperm into the female genital tract and are therefore invariably subfertile. In this review, the pathophysiology, diagnosis and treatment of AE and RE are discussed.

\section{EJACULATORY PHYSIOLOGY}

During sexual activity, rising levels of arousal reach a threshold that triggers the ejaculatory response. Rhythmic contractions of the ischiocavernosus and bulbospongiosus muscles as well as semen expulsion are mediated by sensory neurons in the pelvis. These events are associated with the occurrence of orgasmic climax, a distinct cortical event that is experienced both cognitively and emotionally. The latency to ejaculation, i.e. the time and degree of stimulation required for ejaculation, varies significantly among men and even for individual men in different situations. While most men and their partners are satisfied with the latency of their ejaculatory response, many others are dissatisfied. ${ }^{5,6}$

The ejaculatory reflex is a complex neurobiological event, involving a coordination of actions in the central and peripheral nervous systems to bring about synchronized neurological and muscular responses. ${ }^{2}$ Spinal ejaculatory centers receive input from supraspinal regions ( $\mathrm{cog}$ nitive arousal), as well as from peripheral sensory afferents from the genitals. ${ }^{5}$ A spinal control center, referred to as a spinal pattern generator, coordinates sympathetic, parasympathetic and motor (somatic) outflows, integrating the latter with the inputs from the supraspinal sites in the brainstem, hypothalamus and preoptic area. The subsequent ejaculatory process is typically divided into two distinct phases that occur in sequence: emission and expulsion. ${ }^{6}$

The first of these phases, emission, is initiated by the thoracolumbar sympathetic nerves, which exit the spinal cord at the levels T10-L2. These nerves initiate peristaltic contraction of smooth muscle in the epididymis, vas deferens, seminal vesicles and prostate. These coordinated actions propel sperm through the male excurrent ductal system into the ejaculatory ducts and urethra. Contractions of smooth muscle within the seminal vesicles simultaneously propel seminal fluid through the ejaculatory ducts. Ultimately, a bolus of sperm, seminal fluid and prostatic fluid are deposited into the posterior urethra. Forceful contraction of the bladder neck prevents ejaculate from flowing retrograde into the bladder. ${ }^{5,7}$ 
Distention of the posterior urethra by the seminal fluid bolus stimulates a reflex arc that triggers the second phase of ejaculation, expulsion. The pudendal nerve, which originates from the sacral spinal cord (S2-S4), mediates involuntary rhythmic contractions of striated peri-urethral (ischiocavernosus and bulbospongiosus) and pelvic floor muscles. Meanwhile, relaxation of the external urinary sphincter occurs. The end result is rhythmic, forcible ejection of seminal fluid from the posterior urethra towards the urethral meatus. ${ }^{7}$

Failure of the initial step, emission, leads to AE. Inadequate bladder neck closure leads to failure of the second step, expulsion, which defines RE. In contrast to PE and DE, which may arise from either psychogenic or physiological mechanisms and can sometimes be managed by behavorial modification, AE and RE predominantly result from organic neurogenic or anatomic dysfunction. ${ }^{5}$ Ejaculatory dysfunction of all types, however, may result from psychogenic etiology. Psychogenic ejaculatory dysfunction is a diagnosis of exclusion and its management is beyond the scope of this paper.

\section{ANEJACULATION (AE)}

$\mathrm{AE}$ is a relatively rare condition but represents an important causative factor of male subfertility. The causes of $\mathrm{AE}$ can be categorized as organic, pharmacological and psychogenic. Any disease, surgical procedure, or trauma that interferes with the afferent or efferent innervation of the seminal vesicles, vasa deferentia, bladder neck, or posterior urethra can potentially result in AE. Organic etiologies of AE include diabetes mellitus (DM), spinal cord injury (SCI), transverse myelitis, multiple sclerosis and surgical injury of the autonomic nervous system. ${ }^{8}$ Ejaculatory duct obstruction may also present with reduced or absent semen volume. It represents, however, an entity distinct from AE. Psychogenic AE has been associated with anhedonia, as well as anxiety-related specifically to ejaculation. ${ }^{8,9}$ The diagnosis of psychogenic AE remains one of exclusion and can only be made after ruling out biological etiologies.

The most common causes of $\mathrm{AE}$ are retroperitoneal lymph node dissection (RPLND) and SCI, together accounting for almost $90 \%$ of cases. ${ }^{8,10}$ RPLND is performed in men with testis cancer in which retroperitoneal nodal tissue is resected from around the great vessels to remove known or potential sites of cancer metastasis. AE results from injury to the sympathetic chains or postganglionic branches of the lumbar sympathetic nerves. Fortunately, the widespread use in recent years of modified templates and nerve-sparing techniques have allowed for excellent preservation of antegrade ejaculation without compromising oncological outcomes. A review of 176 men between 3 and 9 years after primary RPLND at Indiana University demonstrated that $99 \%$ of patients retained the ability to ejaculate after a nerve-sparing procedure. ${ }^{11}$ Antegrade ejaculatory function remains threatened, however, for men in whom nerve-sparing techniques are not carefully performed or when nerve sparing must be sacrificed for oncological control. It is often difficult to spare nerves, most notably, in patients undergoing postchemotherapy RPLND. In these cases, extensive retroperitoneal fibrosis often presents a formidable barrier to nerve sparing. Various series of patients undergoing postchemotherapy RPLND have been described, with postoperative antegrade ejaculation rates reported to be below $80 \% .^{12}$

SCI patients with upper motor neuron lesions above spinal cord level T9 frequently experience some degree of erectile impairment. It is the complete absence of the ejaculatory reflex, however, that represents the most significant barrier to fertility in these patients. ${ }^{13}$ The combination of erectile dysfunction and ejaculatory dysfunction renders approximately $95 \%$ of SCI patients unable to procreate without medical intervention. ${ }^{14}$

DM is a well-known cause of autonomic neuropathy, affecting numerous peripheral nerves including those responsible for both erectile and ejaculatory function. The manifestations of diabetic neuropathy on ejaculatory function vary, but most commonly present as RE secondary to incomplete bladder neck closure. More severe cases of diabetic neuropathy are associated with absence of vasal and seminal vesicle peristalsis and resultant failure of seminal emission. While the incidence of RE in male diabetics has been estimated at $6 \%$, the true incidence of clinically significant $\mathrm{AE} / \mathrm{RE}$ in patients with $\mathrm{DM}$ is unknown, because many older men who develop DM are uninterested in fertility and therefore go unrecognized. ${ }^{15}$ The overall prevalence of DM among men undergoing evaluation for infertility is low and ranges from $0.7 \%$ to $1.1 \%$. $^{16,17}$

In addition to the medical and surgical causes of ejaculatory dysfunction, a number of medications have been shown to interfere with ejaculation. At the forefront are psychotropic drugs, which have some of the highest reported rates of associated sexual dysfunction. This group includes thioridazine, isocarboxazid, phenelzine, fluphenazine, trifluoperazine and tranylcypromine. ${ }^{18}$ Selective serotonin reuptake inhibitors and serotonin norepinephrine reuptake inhibitors have also been associated with ejaculatory problems; in fact, $73 \%$ of patients taking these medications report sexual difficulties, with two-thirds complaining of orgasmic dysfunction. ${ }^{19}$

Use of $\alpha$-1-adrenoreceptor antagonists is also associated with abnormal ejaculation. These medications relieve obstructive urinary symptoms by relaxing $\alpha$-1-adrenoreceptors present in the smooth muscle of the prostate, urethra and bladder neck. This mechanism of action is also responsible for undesirable effects on ejaculation. By relaxing smooth muscle in the bladder neck, vasa defentia and seminal vesicles, $\alpha$-blockers result in ejaculatory dysfunction in anywhere from $4 \%$ to $11 \%$ of patients, depending on their degree of selectivity for the $\alpha$-1A receptor (the most prominent subtype found in the bladder neck and prostate). ${ }^{20}$ The $\alpha-1 \mathrm{~A}$ selective receptor antagonist tamsulosin, for example, has been shown to reduce ejaculate volume in $90 \%$ of healthy volunteers, as compared to only $21 \%$ of men taking alfuzosin (a non-selective $\alpha$-1-adrenoreceptor antagonist). Moreover, tamsulosin has a reported AE rate of 35\% as compared to $0 \%$ for alfuzosin. ${ }^{21}$

\section{RETROGRADE EJACULATION (RE)}

$\mathrm{RE}$ is a common type of ejaculatory dysfunction, but accounts for only $0.3 \%-2 \%$ of male infertility. ${ }^{22,23}$ This condition is marked by substantial retrograde propulsion of seminal fluid into the bladder. In the normal physiological state, the bladder neck closes with high pressure during ejaculation, forcing semen deposited into the posterior urethra to flow antegrade through the urethra and out the urethral meatus. With impaired or absent bladder neck closure, however, semen in the proximal urethra may be propelled along the path of least resistance into the bladder.

The presumptive diagnosis of RE is often reached by taking a careful patient history with attention to coexisting medical conditions, medications and surgical history. In men who present with absent or lowvolume ejaculate, RE and ejaculatory duct obstruction comprise the differential diagnosis. Patients with RE frequently describe a cloudy appearance of postorgasmic urine. The diagnosis of RE is confirmed by a post-ejaculatory voided urine that reveals spermatozoa, seminal fluid or fructose. ${ }^{2,24}$ 
The underlying causes of RE may be divided into pharmacological, neurogenic and anatomic etiologies. Pharmacologically-induced RE, as previously discussed, is largely attributable to psychotropic medications and $\alpha$-adrenergic blockers. ${ }^{10}$ Neurogenic causes include spinal cord lesions, retroperitoneal surgery (including lumbar sympathectomy, RPLND, aortoiliac vascular surgery and abdomino-perineal resection), and neuropathies (such as diabetic autonomic neuropathy and multiple sclerosis).

Since closure of the bladder neck is under sympathetic control, medications that inhibit closure of the bladder neck can reversibly cause RE. Anatomic etiologies can be subdivided into congenital (posterior urethral valves, utricular cysts and exstrophy) or acquired conditions. Acquired conditions are far more common and usually result from mechanical disruption of the bladder neck and/or prostate. For example, $30 \%$ and $41 \%$ of men report new-onset RE following transurethral vaporization of the prostate and transurethral resection of the prostate, respectively. ${ }^{25,26}$

Despite the numerous causes of RE, four etiologies collectively account for more than $80 \%$ of cases. These factors are DM, prior history of RPLND, bladder neck surgery and transurethral resection of the prostate. ${ }^{10}$

\section{PHARMACOLOGICAL TREATMENT OF RE AND AE}

Medical treatment of RE and AE is less invasive than procedural interventions and offers patients the possibility of naturally conceiving children. $\alpha$-blockers and antidepressants should be discontinued, if possible, to observe for improvement in ejaculatory dysfunction. Just as $\alpha$-blockade can lead to RE or AE, the administration of sympathomimetic agents may help to reverse these abnormalities in patients with ejaculatory dysfunction. ${ }^{8}$ Thus, pharmacological treatment is therefore considered first-line therapy for patients with ejaculatory dysfunction. The efficacy of this approach largely depends on the etiology of ejaculatory dysfunction. Structural causes, as in patients with prior histories of bladder neck or prostatic resection, are generally irreversible and not amenable to pharmacological treatment.

Neurological causes of AE and RE, such as SCI, are often similarly refractory to medical therapy. However, in some patients with partial neurological injuries (i.e. in the early stages of progressive neurological diseases or with incomplete peripheral lesions following surgery or trauma) several classes of medications have been used to successfully induce antegrade ejaculation. These include $\alpha$-adrenergic agonists, anticholinergics and antihistaminic drugs which function either by increasing the sympathetic or decreasing the parasympathetic tone of the bladder neck. ${ }^{10,27}$ These agents facilitate antegrade propulsion of semen by increasing bladder neck tone.

The efficacy of pharmacotherapy for the treatment of RE has been evaluated in several studies. A meta-analysis including 264 patients who were treated with $\alpha$-agonists, anticholinergics, and antihistamines for reversal of RE reported a $50 \%$ success rate as defined by induction of antegrade ejaculation. Moreover, 32/95 (34\%) of patients with a documented interest in fertility were able to achieve a spontaneous pregnancy during treatment. The most commonly used drug was imipramine, which accounted for $80 \%$ of the treatment where pregnancy was desired and $94 \%$ of the spontaneous pregnancies achieved. ${ }^{10}$ Imipramine is a tricyclic with $\alpha$-adrenergic activity that has been shown to cause an increase in the pressure profile of the posterior urethra. ${ }^{28}$

Ochsnenkuh et al. ${ }^{29}$ evaluated 11 patients with iatrogenic RE (secondary to RPLND in 10 and aortic surgery in 1). All patients received $25 \mathrm{mg}$ imipramine daily for 4 days, followed by $50 \mathrm{mg}$ daily. The authors found that imipramine induced antegrade ejaculation in all 11 patients, and two spontaneous pregnancies were reported within 6 months of initiating treatment. ${ }^{29}$ Despite the impressive results yielded in this study, the published success rates for restoring antegrade ejaculation vary from $0 \%$ to $100 \%$, with an average success rate across studies reported to be $46 \%$ for reversal of RE. ${ }^{30}$ Given the intermittent success rates and limited adverse effects profile, it has been argued that 25-75 mg per day of oral imipramine should be applied as a first-line agent in patients with RE. ${ }^{10}$

Several $\alpha$-adrenergic agonists have also been widely used for the treatment of both RE and AE. These sympathomimetics, including pseudoephedrine, ephedrine and midodrine, are alternative pharmacological choices to imipramine. They each function similarly by increasing bladder neck tone but, like imipramine, are far more successful in reversing RE compared to AE. Overall, $\alpha$-agonists have been reported to produce antegrade ejaculation in $50 \%$ of men with RE, but only in $12 \%$ of patients with AE. Exceedingly few cases of spontaneous pregnancies in partners of patients with $\mathrm{AE}$ after treatment with $\alpha$ agonistics have been reported. ${ }^{30}$

Midodrine is an $\alpha$-adrenergic receptor agonist primarily used to treat orthostatic hypotension. It has also been shown to stimulate sympathetically innervated structures, including the vasa deferentia, prostate and seminal vesicles, and to promote the rhythmic muscle contraction required during ejaculation. In a recent study, 185 SCI patients who failed to respond to penile vibratory stimulation (PVS) were treated with midodrine prior to repeat stimulation. Antegrade ejaculation or RE subsequently occurred in $64.6 \%$ of the patients. ${ }^{31}$ In another study that included 62 men with SCI, $22 \%$ of men that did not respond to PVS alone were salvaged by midodrine combined with PVS. $^{32}$

The use of midodrine is not limited to patients with SCI, as illustrated by a placebo-controlled study evaluating the efficacy of midodrine for the treatment of organic AE without SCI. In this study midodrine induced antegrade, retrograde and antegrade, and RE in $29.5 \%, 13.1 \%$ and $14.8 \%$ of patients, respectively, thereby reversing AE in more than half of patients. ${ }^{9}$ The overall success rate (defined as antegrade or RE) of midodrine in $\mathrm{AE}$ patients has been reported to be as high as $61 \%$, which is significantly higher than that of pseudoephidrine or ephedrine. ${ }^{10}$

Other studies have suggested that combination therapy is superior to treatment with a single agent. For example, in a study of 33 diabetic men with RE ( 23 complete and 10 partial), antegrade ejaculation was successfully achieved in $48 \%$ of men taking pseudoephedrine, $39 \%$ of men on imipramine and in $62 \%$ of patients administered both pseudoephedrine and imipramine. ${ }^{33}$

\section{ASSISTED EJACULATION PROCEDURES}

In men who fail pharmacological conversion to antegrade ejaculation or in whom pharmacological management is contraindicated, assisted ejaculation procedures may be utilized to harvest sperm. If RE cannot be reversed pharmacologically, the next step is post-ejaculatory harvesting of sperm in the bladder. ${ }^{27}$ Urinary sperm retrieval is achieved by alkalinizing the urine using oral sodium bicarbonate, catheterizing to obtain a post-ejaculatory urine specimen and then centrifuging and resuspending the specimen in medium before using it for vaginal or intrauterine insemination. ${ }^{34}$ A suggested protocol is presented in Table 1.

For men who fail medical management of AE, sperm retrieval can be attempted using either PVS or electroejaculation (EEJ). If no viable sperm can be retrieved with these methods, surgical sperm retrieval 


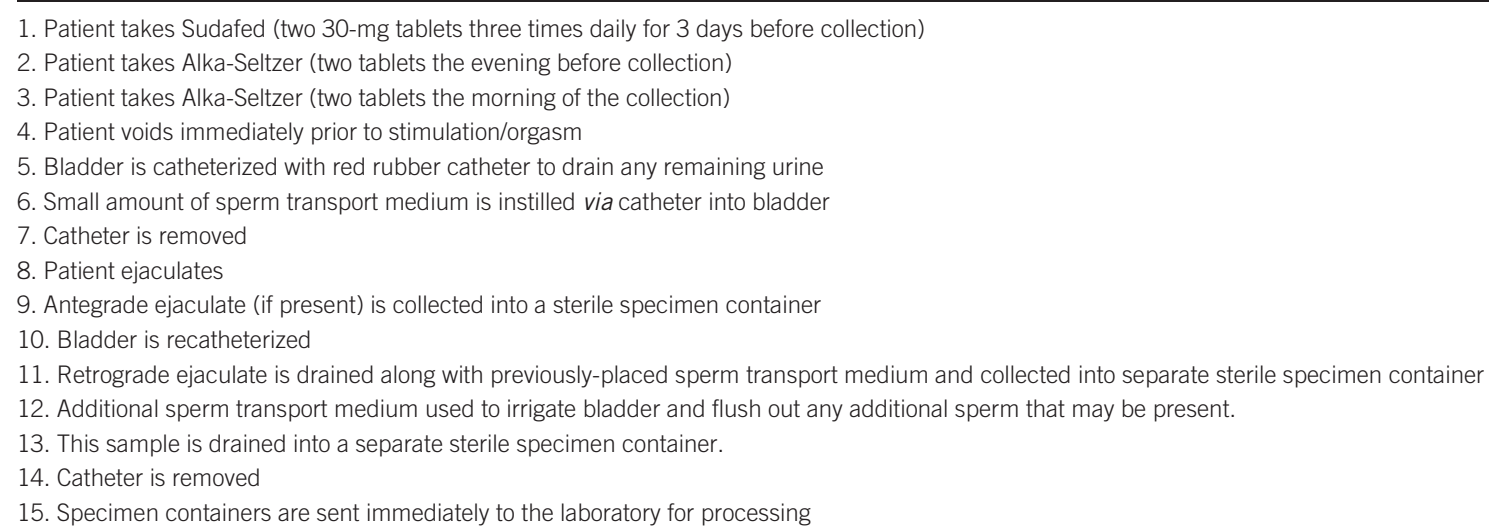

may be performed using microsurgical epididymal sperm aspiration, percutaneous epididymal sperm aspiration, testicular sperm aspiration, or testicular sperm extraction. These modalities enable acquisition of sperm for intracytoplasmic sperm injection and for cryopreservation of sperm in azoospermic men desiring assisted reproduction in the future. ${ }^{10,35}$

\section{PENILE VIBRATORY STIMULATION (PVS)}

PVS is an effective, well-tolerated, office-based procedure that involves placing a vibrator in the perifrenular area of the glans penis for several minutes or until antegrade ejaculation occurs. With the exception of infrequent mild skin abrasions at the site of stimulator application, adverse effects are uncommon. ${ }^{8}$

One notable exception, however, is the dangerous potential for autonomic dysreflexia $(\mathrm{AD})$ to occur in association with PVS. $\mathrm{AD}$ is a syndrome of large magnitude reflex sympathetic discharge associated with dangerously high systemic blood pressure that can induce seizure, stroke, or death. These reflexively evoked increases in sympathetic vasoconstrictor drive have been documented in up to $70 \%$ of men with high spinal cord injuries. ${ }^{36}$ For at-risk SCI patients (those with lesions above T6), pre-treatment with nifedipine is useful in ablating the response and constant monitoring of vital signs during the procedure is essential. Moreover, spinal or general anesthesia is highly effective at averting $\mathrm{AD}$ and should be strongly considered in patients at risk. ${ }^{37}$

For PVS to be successful, patients need an intact ejaculatory reflex arc. This requires that the sensory afferents from the penis, the spinal cord at the levels of sensory input (S2-S4), the efferent exit (T10-L2), and sympathetic outflow must all be functioning. Therefore, optimal candidates for PVS are SCI patients with lesions above T10; in this group, PVS produces ejaculation in $88 \%$ of men. ${ }^{38}$ In patients with spinal cord injuries below T10, on the other hand, ejaculatory success rates are as low as $15 \%{ }^{39}$

In an effort to identify the optimal settings, Sonksen et al. ${ }^{38}$ evaluated the ejaculatory response to PVS in men with SCI. The authors found that a vibrator amplitude level of $2.5 \mathrm{~mm}$ and a frequency of $100 \mathrm{~Hz}$ produced antegrade and/or RE in $96 \%$ of subjects, compared to $32 \%$ when the amplitude was $1 \mathrm{~mm}$ with a frequency of $100 \mathrm{~Hz}$. These results led to the development of a medical grade vibrator for PVS called FertiCare Personal (Orion Medical Group, Silverado, CA, USA) that is the first FDA-approved device for ejaculation induction in men with SCI.

\section{ELECTROEJACULATION (EEJ)}

EEJ is an alternative approach to PVS with similar medical indications. With this approach, a specifically designed electric probe that is connected to an adjustable output power source is gently inserted into the rectum. General anesthesia is required except in some cases of complete spinal cord injuries where painful sensation from the insertion and action of the probe is not an issue. $\mathrm{AD}$ is a potential side effect with EEJ, as well as with PVS. As discussed previously, in order to prevent $\mathrm{AD}$ in men with SCI above the level of $\mathrm{T} 6$, prophylactic medication with nifedipine just prior to the procedure is prudent. ${ }^{8}$

Rhythmic delivery of current is performed by manually turning the dial to increase the voltage delivery progressively for a few seconds at each level. Voltage is increased until ejaculation occurs. ${ }^{8}$ Partial erection is often, but not always, induced by the action of the EEJ probe. EEJ may induce ejaculation that is entirely retrograde. In these cases, diaphoresis, piloerection and penile erection may be the only signs that ejaculation has occurred and that sperm may be retrieved from the bladder. Immediately after the rectal probe has been removed, a red rubber catheter should be placed in the bladder and flushed with sperm transport medium so that any sperm in the bladder may be drained into a sterile collection container.

Successful retrieval of sperm has been reported in $97 \%$ of patients treated with EEJ. ${ }^{40}$ In a review of 28 men with SCI, an overall ejaculation rate of $100 \%$ was achieved ( $79 \%$ via PVS and the remaining $21 \%$ via EEJ). Using EEJ in combination with assisted reproductive techniques, including vaginal self-insemination, intrauterine insemination, in vitro fertilization and intracytoplasmic sperm injection, nearly one-third of the couples (9/28) were shown to achieve pregnancy. ${ }^{41}$ Thus, EEJ presents an effective treatment option for men with ejaculatory dysfunction.

\section{CONCLUSIONS}

Ejaculatory dysfunction is a common and distressing cause of male sexual bother. It also represents a significant cause of male factor subfertility, posing distinct reproductive challenges for couples attempting to conceive. $\mathrm{AE}$ and $\mathrm{RE}$ may result from pharmacological, neurogenic or anatomical causes. Pharmocological treatments are a first-line therapy and have mixed results. Post-ejaculatory urine collection provides a minimally invasive alternative for men with RE and desiring fertility. However, for men with AE, more invasive assisted ejaculation techniques, such as PVS or EEJ, are considered second-line therapy but have very high and predictable success rates for inducing ejaculation. When these measures fail, surgical sperm retrieval using microsurgical epididymal sperm aspiration, percutaneous epididymal sperm aspiration, testicular sperm aspiration, or testicular sperm extraction may be performed in combination with intracytoplasmic sperm injection to achieve pregnancy. Thus, men with AE and RE can 
expect to achieve conception with excellent success rates given the wide range of therapeutic modalities available to them.

\section{COMPETING FINANCIAL INTERESTS}

The authors declare no competing financial interests.

1 Rosen R, Altwein J, Boyle P, Kirby RS, Lukacs B et al. Lower urinary tract symptoms and male sexual dysfunction: the multinational survey of the aging male (MSAM-7). Eur Urol 2003; 44: 637-49.

2 Rowland D, McMahon CG, Abdo C, Chen J, Jannini E et al. Disorders of orgasm and ejaculation in men. J Sex Med 2010; 7: 1668-86.

3 Corona G, Jannini EA, Lotti F, Boddi V, De Vita G et al. Premature and delayed ejaculation: two ends of a single continuum influenced by hormonal milieu. Int $J$ Androl. 2011; 34: 41-8.

4 Shindel AW, Nelson CJ, Naughton CK, Mulhall JP. Premature ejaculation in infertile couples: prevalence and correlates. J Sex Med 2008; 5: 485-91.

5 Betocchi C, Verze P, Palumbo F, Arcaniolo D, Mirone V. Ejaculatory disorders: pathophysiology and management. Nat Clin Pract Urol 2008; 5: 93-103.

6 Calabrò RS, Polimeni G, Ciurleo R, Casella C, Bramanti P. Neurogenic ejaculatory disorders: focus on current and future treatments. Recent Pat CNS Drug Discov 2011; 6: 205-21.

7 Hershlag A, Schiff SF, DeCherney AH. Retrograde ejaculation. Hum Reprod 1991; 6 : 255-8.

8 OhI DA, Quallich SA, Sønksen J, Brackett NL, Lynne CM. Anejaculation and retrograde ejaculation. Urol Clin North Am 2008; 35: 211-20.

9 Safarinejad MR. Midodrine for the treatment of organic anejaculation but not spina cord injury: a prospective randomized placebo-controlled double-blind clinical study. Int J Impot Res 2009; 21: 213-20.

10 Kamischke A, Nieschlag E. Update on medical treatment of ejaculatory disorders. Int J Androl 2002; 25: 333-44.

11 Beck SD, Bey AL, Bihrle R, Foster RS. Ejaculatory status and fertility rates after primary retroperitoneal lymph node dissection. J Urol 2010; 184: 2078-80.

12 Coogan CL, Hejase MJ, Wahle GR, Foster RS, Rowland RG et al. Nerve sparing postchemotherapy retroperitoneal lymph node dissection for advanced testicular cancer. J Urol 1996; 156: 1656-8.

13 Biering-Sørensen F, Sønksen J. Sexual function in spinal cord lesioned men. Spina Cord 2001; 39: 455-70.

14 Ohl DA, Quallich SA, Sonksen J, Brackett NL, Lynne CM. Anejaculation: an electrifying approach. Semin Reprod Med 2009; 27: 179-85.

15 Dinulovic D, Radonjic G. Diabetes mellitus/male infertility (review). Arch Androl 1990; 25: 277-93.

16 Greenberg SH, Lipshultz LI, Wein AJ. Experience with 425 subfertile male patients. J Urol 1978; 119: 507-10

17 Sexton WJ, Jarow JP. Effect of diabetes mellitus upon male reproductive function. Urology 1997; 49: 508-13.

18 Shiloh R. Drugs affecting sexual function. In: Atlas of Psychiatric Pharmacotherapy. 2nd ed. London: Taylor \& Francis; 2006. pp105-13.

19 Clayton AH, Balon R. The impact of mental illness and psychotropic medications on sexual functioning: the evidence and management. J Sex Med 2009; 6: 1200-11.
20 Höfner K, Claes H, De Reijke TM, Folkestad B, Speakman MJ. Tamsulosin 0.4 mg once daily: effect on sexual function in patients with lower urinary tract symptoms suggestive of benign prostatic obstruction. Eur Urol 1999; 36: 335

21 Hellstrom WJ, Sikka SC. Effects of acute treatment with tamsulosin versus alfuzosin on ejaculatory function in normal volunteers. J Urol 2006; 176: 1529-33.

22 Vernon M, Wilson E, Muse K, Estes S, Curry T. Successful pregnancies from men with retrograde ejaculation with the use of washed sperm and gamete intrafallopian tube transfer (GIFT). Fertil Steril 1988; 50: 822-4.

23 Yavetz H, Yogev L, Hauser R, Lessing JB, Paz G et al. Retrograde ejaculation. Hum Reprod 1994; 9: 381-6.

24 Colpi G, Weidner W, Jungwirth A, Pomerol J, Papp G et al. EAU guidelines on ejaculatory dysfunction. Eur Uro/ 2004; 46: 555-8.

25 Spaliviero M, Strom KH, Gu X, Araki M, Culkin DJ et al. Does Greenlight HPS ${ }^{\text {TM }}$ lase photoselective vaporization prostatectomy affect sexual function? J Endourol 2010: 24: 2051-7.

26 Hill B, Belville W, Bruskewitz R, Issa M, Perez-Marrero R et al. Transurethral needle ablation versus transurethral resection of the prostate for the treatment of symptomatic benign prostatic hyperplasia: 5-year results of a prospective, randomized, multicenter clinical trial. J Urol 2004; 171: 2336

27 Fode M, Krogh-Jespersen S, Brackett NL, OhI DA, Lynne CM et al. Male sexual dysfunction and infertility associated with neurological disorders. Asian J Androl $2011 ; 14: 61-8$

28 Goldwasser B, Madgar I, Jonas P, Lunefeld B, Many M. Imipramine for the treatment of sterility in patients following retroperitoneal lymph node dissection. Andrologia 1983; 15: 588-591.

29 Ochsnenkuh R, Kamischke A, Nieschlag E. Imipramine for successful treatment of retrograde ejaculation caused by retroperitoneal surgery. Int J Androl 1999; 22: 173-7.

30 Kamischke A, Nieschlag E. Treatment of retrograde ejaculation and anejaculation. Hum Reprod Update 1999; 5: 448-74.

31 Soler JM, Previnaire JG, Plante P, Denys P, Chartier-Kastler E. Midodrine improves ejaculation in spinal cord injured men. J Urol 2007; 178: 2082-6.

32 Courtois FJ, Charvier KF, Leriche A, Vézina JG, Côté M et al. Blood pressure changes during sexual stimulation, ejaculation and midodrine treatment in men with spinal cord injury. BJU Int 2008; 101: 331-7.

33 Arafa M, El Tabie O. Medical treatment of retrograde ejaculation in diabetic patients: a hope for spontaneous pregnancy. J Sex Med 2008; 5: 194-8.

34 Jefferys $A$, Siassakos D, Wardle $P$. The management of retrograde ejaculation: a systematic review and update. Fertil Steril 2011; 97: 306-12.

35 Wein AJ, Kavoussi LR, Novick AC, Partin AW, Peters CA, editors. Campbell-Walsh Urology. 10th ed. Chapter 22: Surgical Management of Male Infertility. PhiladelphiaPA: Saunders; 2011.

36 Brown R, Stolzenhein G, Engel S, Macefield VG. Cutaneous vasoconstriction as a measure of incipient autonomic dysreflexia during penile vibratory stimulation in spinal cord injury. Spinal Cord 2009; 47: 538-44.

37 Brackett NL, Lynne CM, Ibrahim E, OhI DA, Sønksen J. Treatment of infertility in men with spinal cord injury. Nat Rev Urol 2010; 7: 162-72.

38 Sønksen J, Ohl DA. Penile vibratory stimulation and electroejaculation in the treatment of ejaculatory dysfunction. Int J Androl 2002; 25: 324-32.

39 Kafetsoulis A, Brackett NL, Ibrahim E, Attia GR, Lynne CM. Current trends in the treatment of infertility in men with spinal cord injury. Fert Steril 2006; 86: 781-9.

40 Iremashvili V, Brackett NL, Ibrahim E, Aballa TC, Lynne CM. The choice of assisted ejaculation method is relevant for the diagnosis of azoospermia in men with spinal cord injuries. Spinal Cord 2011; 49: 55-9.

41 Sønksen J, Sommer P, Biering-Sørensen F, Ziebe S, Lindhard A et al. Pregnancy after assisted ejaculation procedures in men with spinal cord injury. Arch Phys Med Rehabil 1997; 78: 1059-61. 